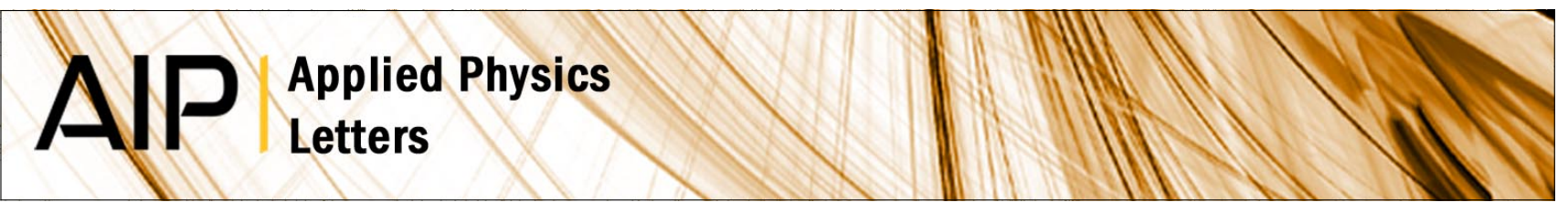

\title{
Many-body effects in InAs/GaAs quantum dot laser structures
}

I. O’Driscoll, M. Hutchings, P. M. Smowton, and P. Blood

Citation: Appl. Phys. Lett. 97, 141102 (2010); doi: 10.1063/1.3496011

View online: http://dx.doi.org/10.1063/1.3496011

View Table of Contents: http://apl.aip.org/resource/1/APPLAB/v97/i14

Published by the American Institute of Physics.

\section{Related Articles}

Electroluminescence from strained germanium membranes and implications for an efficient Si-compatible laser Appl. Phys. Lett. 100, 131112 (2012)

A weakly coupled semiconductor superlattice as a potential for a radio frequency modulated terahertz light emitter

Appl. Phys. Lett. 100, 131104 (2012)

Quantum-dot nano-cavity lasers with Purcell-enhanced stimulated emission

Appl. Phys. Lett. 100, 131107 (2012)

Effect of internal optical loss on the modulation bandwidth of a quantum dot laser

Appl. Phys. Lett. 100, 131106 (2012)

Design of three-well indirect pumping terahertz quantum cascade lasers for high optical gain based on nonequilibrium Green's function analysis

Appl. Phys. Lett. 100, 122110 (2012)

\section{Additional information on Appl. Phys. Lett.}

Journal Homepage: http://apl.aip.org/

Journal Information: http://apl.aip.org/about/about_the_journal

Top downloads: http://apl.aip.org/features/most_downloaded

Information for Authors: http://apl.aip.org/authors

\section{ADVERTISEMENT}

\section{ACCELERATE AMBER AND NAMD BY $5 X$.}

NVIDIA TRYIT ONA FREE, REMOTELYYHOSTED CLUSTER. 


\title{
Many-body effects in InAs/GaAs quantum dot laser structures
}

\author{
I. O'Driscoll, ${ }^{\text {a) }}$ M. Hutchings, P. M. Smowton, and P. Blood \\ Cardiff School of Physics and Astronomy, Cardiff University, Queens Buildings, Cardiff CF24 3AA, \\ United Kingdom
}

(Received 23 July 2010; accepted 10 September 2010; published online 4 October 2010)

\begin{abstract}
We have measured the gain peak energy of GaInAs quantum dot laser structures, relative to the absorption peak, as a function of injection. We have used a calculation to remove the effects of state filling in the inhomogeneous distribution and to estimate the carrier density in the dots. We have identified shifts, which we associate with many body effects, of up to $8 \mathrm{meV}$ at room temperature at injection levels typical for laser operation of about 2.2 electrons per dot, producing a peak modal gain of $10 \mathrm{~cm}^{-1}$. (C) 2010 American Institute of Physics. [doi:10.1063/1.3496011]
\end{abstract}

It has long been recognized that the optical spectra of self-assembled quantum dot laser structures are strongly influenced by homogeneous broadening arising from dephasing due to carrier-carrier interactions. ${ }^{1}$ However it is also apparent from experimental studies ${ }^{2}$ that under excitation the gain peak moves to lower photon energy, and in some cases gain is observed at photon energies where there is no absorption, which is important in the design of quantum dot vertical cavity lasers. Theoretical studies of the optical properties of semiconductor microcrystallites ${ }^{3}$ suggested that where strong confinement is imposed in all three directions there is no many-body renormalization of the transition energy, while later theoretical work suggests there may be significant deviation from this simple situation. ${ }^{4}$ Nair and Masumoto ${ }^{5}$ have calculated a reduction in the transition energy of typical self-assembled dots due to Coulomb interactions of up to about $20 \mathrm{meV}$ for 18 electron-hole (e-h) pairs per dot. Single dot photoluminescence (PL) experiments of Matsuda et al. ${ }^{6}$ at $300 \mathrm{~K}$ are consistent with this, showing a reduction in transition energy of about $6 \mathrm{meV}$ for $2.5 \mathrm{e}-\mathrm{h}$ pairs per dot. Heitz et al. ${ }^{7}$ report shifts from PL measurements at $7 \mathrm{~K}$ of only $1.5 \mathrm{meV}$ at $3 \mathrm{e}$-h pairs per dot but rising to $15 \mathrm{meV}$ at 18 e-h pairs per dot. Additionally, Schneider and $\mathrm{Chow}^{8}$ have shown theoretically that the Coulomb interaction with the carriers in the continuum states associated with the adjacent well or wetting layer can produce redshifts in the gain spectrum of $20 \mathrm{meV}$ at carrier densities in the region of $15 \mathrm{e}-\mathrm{h}$ pairs per dot.

We have studied experimentally the evolution with drive current of optical gain spectra of electrically injected quantum dot laser structures at currents necessary for laser operation. We have used a model calculation to remove the spectral shift due to state filling within the inhomogeneous distribution and to estimate the average dot occupation.

We investigated samples grown from two different wafers. The first wafer (sample A) comprised of five layers of InAs dots each grown in a dot-in-a-well on $\operatorname{In}_{0.15} \mathrm{Ga}_{0.85} \mathrm{As}$ surrounded by a GaAs core and $\mathrm{Al}_{0.45} \mathrm{Ga}_{0.55} \mathrm{As}$ cladding waveguide and grown using "high growth temperature spacer layers," 9 while the second wafer (sample B) differed only in GaAs spacer layer thickness and cladding waveguide composition (see Ref. 10 for further details). The experimental data has been obtained by the electrically pumped, seg-

${ }^{a)}$ Electronic mail: odriscollip@cardiff.ac.uk. mented contact method ${ }^{11}$ and examples of modal gain spectra at various currents at $300 \mathrm{~K}$ are shown in Fig. 1, providing modal gain up to $10 \mathrm{~cm}^{-1}$. These samples contain a bimodal distribution of dots, ${ }^{10,12}$ though this does not have a direct bearing on the results presented here; throughout we focus on the lowest energy gain peak which determines laser action and which is due chiefly to the ground states of the group of "large" dots. The first absorption peak occurs at $0.962 \mathrm{eV}$ whereas the gain peak at the lowest current used (13 mA) appears $14 \mathrm{meV}$ lower.

To analyze these experimental results we require a value for the carrier density but this cannot be measured directly. While it is possible to determine the transparency energy from the gain spectra as an alternative measure of the injection level, this value itself includes the "band gap" and is influenced by the (unknown) shift due to many body interactions. We have used the magnitude of the lowest energy gain peak at each current to estimate the carrier density from a model calculation. The calculation is described in section VI of Ref. 12 and is based on fitting five groups of inhomogeneous transitions, for ground and excited states of "large" and "small" dots, to the absorption spectra. The calculation incorporated homogeneous broadening using a Sech function with a temperature dependent linewidth from Ref. 1 but no other many body interactions. It gives a good description of the spectrally integrated radiative recombination rates and

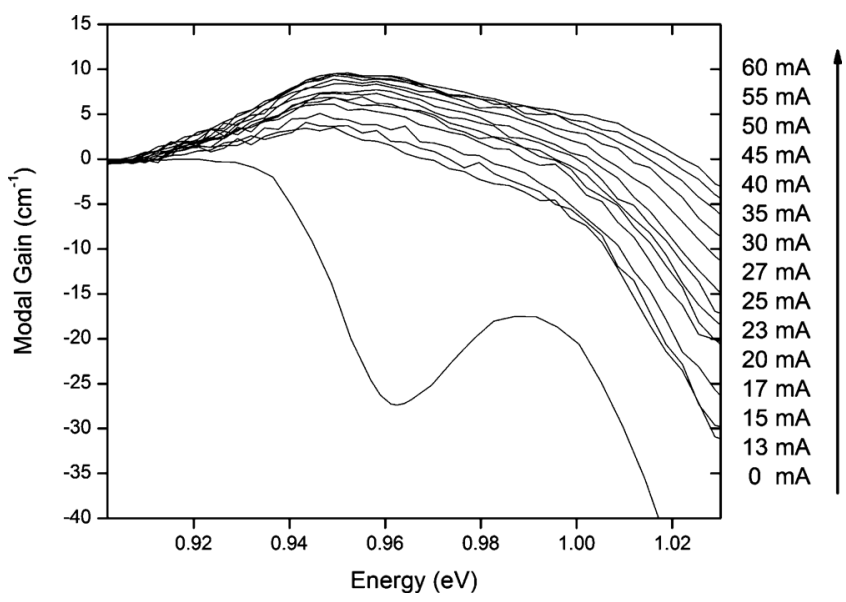

FIG. 1. Plot of modal gain vs energy for pulsed drive currents per section from 13 to $60 \mathrm{~mA}$ at $300 \mathrm{~K}$ for sample $\mathrm{A}$. The modal absorption spectrum is shown as $0 \mathrm{~mA}$. 


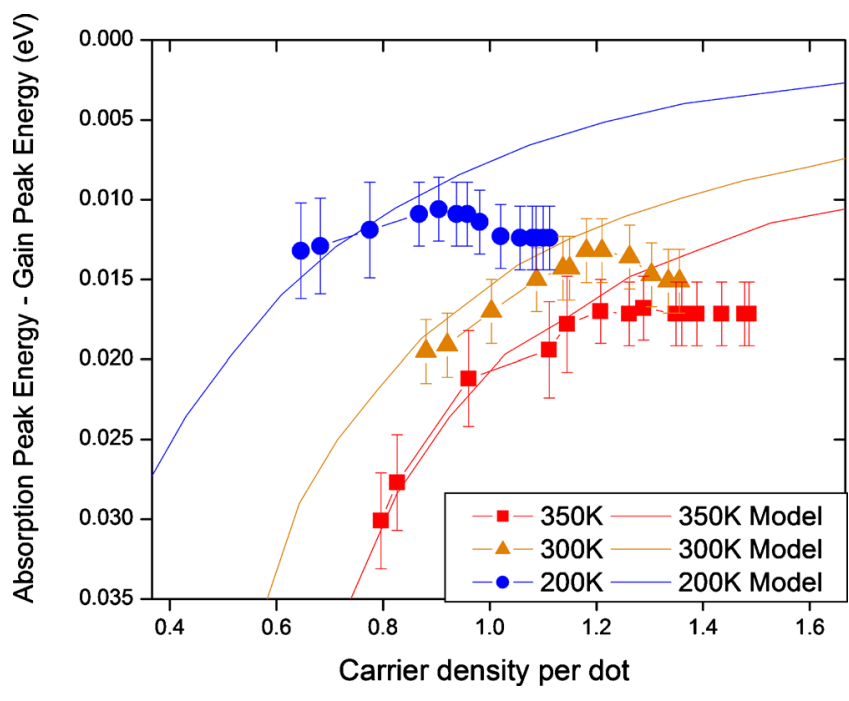

FIG. 2. (Color online) Plot of absorption peak energy minus ground state gain peak energy (from Fig. 1) vs total carrier density per dot (averaged over all dots). The total carrier density per dot is obtained by matching the value of peak gain in the calculation to the experiment for measurements at 200, 300 , and $350 \mathrm{~K}$.

the observed temperature dependence of threshold current. ${ }^{10}$ The dominant ground state contribution to the lowest energy gain peak is given by an expression of the form

$$
G_{p k}=\sigma_{0} N \frac{P(\hbar \varpi)_{p k}}{w_{\text {mod }}}(2 f-1),
$$

where $\sigma_{0}$ is the spectrally integrated optical cross section, $N$ is the dot density, $P(\hbar \varpi)_{p k}$ is the fraction of dots with transition energy at the gain peak in the inhomogeneous distribution, $w_{\text {mod }}$ is the effective mode width, and $f$ is the fractional occupation probability of the ground state [see Ref. 13, Eq. (8)]. The product $\sigma_{0} N$ in the calculation is matched to the area of the corresponding fit to the measured modal absorption spectrum [Eq. (1) with $f=0$ ] so the relation between fractional occupancy of a given group of dots and peak gain does not require knowledge of the dot density.

Figure 2 shows experimental values (as points) for the measured difference between the energy of the lowest absorption peak and the energy of the lowest gain peak (from Fig. 1), plotted as a function of total carrier density per dot (averaged over all dots) obtained as the carrier density necessary in the calculation to produce the magnitude of peak gain in the experiment for measurements at 200, 300, and $350 \mathrm{~K}$. The lines in Fig. 2 are the calculated behavior of the energy of the lowest gain peak, relative to the calculated absorption peak energy, in the absence of many body effects. It is possible that the magnitude of the gain is enhanced by many-body effects, and this could introduce an error into our estimates of the carrier density. However, in the absence of any other measure of the internal injection level, the values in Fig. 2 provide a reasonable basis for taking account of the relative variation with temperature of the carrier density required for a given gain, and for qualitative comparison with other work. We observe that the measured values of gain in Fig. 1, even at the highest currents, do not exceed the magnitude of the measured absorption.

At the lowest injection the experimental gain peak is at lower energy than the absorption peak and is in close agreement with the calculation (Fig. 2) for all temperatures, with

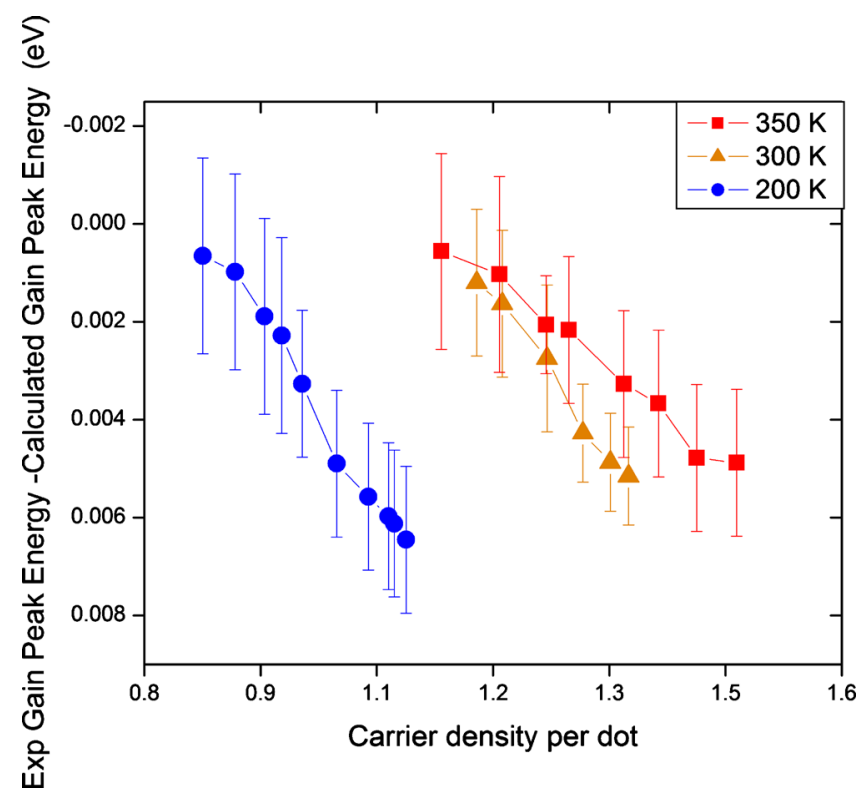

FIG. 3. (Color online) Plot of the energy difference between experiment and calculation, ascribed to many body effects, (obtained from Fig. 2) vs total carrier density per dot. Data is only shown for results when the difference is greater than the experimental error.

no adjustable parameters. This derives from the use of the $\sigma_{0} N$ product from absorption spectra measured directly on these samples, and also suggests negligible gain enhancement due to many body effects, within the experimental uncertainty, in this region. With increasing injection the gain peak moves to higher energy, following the calculation, before deviating from the calculation and moving to lower energies at the highest excitations. We postulate that at very low injection only the lowest energy electron and hole states in the inhomogeneous distribution are populated, producing the initial redshift. Then, as more states are occupied, the gain peak moves to higher energy in the distribution till at the highest injection the experimental gain peak shifts to lower energy due to the increasing effect of many body interactions at high carrier density. The difference between experiment and calculation therefore represents the many-body shift alone and Fig. 3 shows that this difference increases with increasing number of electrons per dot. These results also suggest that the many body shift at a given carrier density may be temperature dependent, decreasing with temperature between 200 and $300 \mathrm{~K}$, however these plots represent the total carrier density, including the higher lying small dots and the wetting layer and the fraction of carriers in these latter states is temperature dependent. Figure 4 shows the shifts for this sample A and for devices from wafer B, as a function of the number of carriers on the large dots divided by the number of large dots, removing the contribution of wetting layer carriers and carriers on the group of small dots which are not colocated with the large dots. Some temperature dependence remains though we note it is smaller in sample B. The magnitudes of the shifts, after the state filling effects have been removed, are $4-8 \mathrm{meV}$ at $300 \mathrm{~K}$ for about 2.2 carriers per dot which is in good agreement with the results from single dot PL measurements ${ }^{6}$ where state filling in an inhomogeneous distribution does not arise.

The calculations of Schneider et al. ${ }^{8}$ for the effects of the wetting layer were done for carrier densities of 3 electrons per dot and above and for a separation of wetting layer and 

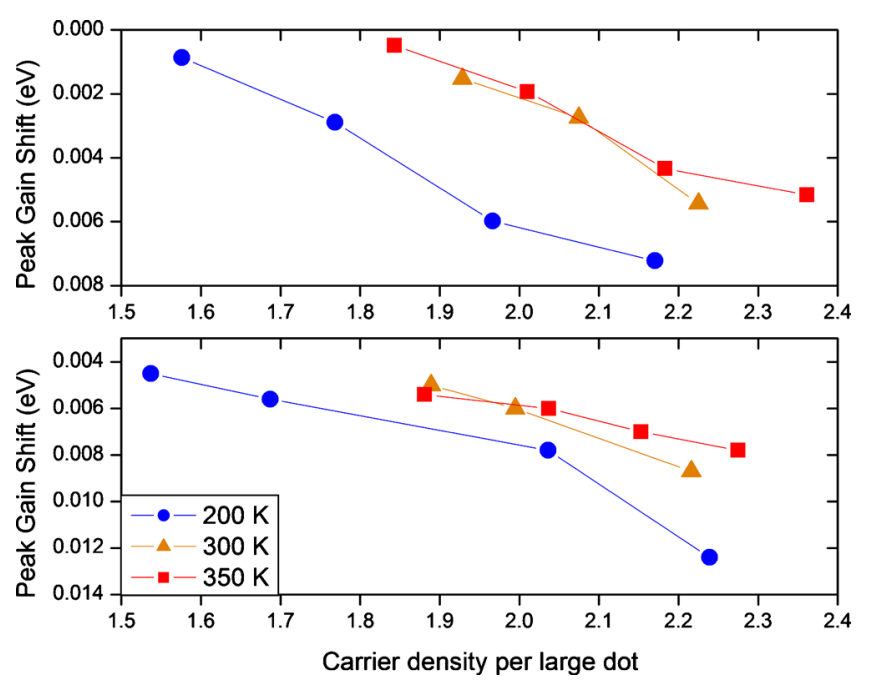

FIG. 4. (Color online) Plot of the difference between experiment and calculation, representing the many body shift, for sample A (top) and sample B (bottom) vs the carrier density per large dot (removing higher lying state contributions and wetting layer states) at 200, 300, and $350 \mathrm{~K}$.

dot transition energies of only $55 \mathrm{meV}$. From photovoltage absorption measurements this energy separation is about 300 $\mathrm{meV}$ in our samples and we estimate from our calculations that for the largest shift at $350 \mathrm{~K}$, the wetting layer carrier density is about 0.065 electrons per dot. We conclude that carriers in the wetting layer do not contribute to the shifts in Fig. 4. Furthermore it was predicted that Coulomb effects decrease the magnitude of the gain at a given carrier density because of differential shifts in the states in the wetting layer and $\operatorname{dot}^{8}$ but we do not expect to see these effects at the injection levels in our experiments.

In conclusion, we have observed the gain peak position of quantum dot structures, relative to the absorption peak, as a function of injection and after removing the effects of state filling in the inhomogeneous distribution we have identified the shifts due to many body effects. The magnitude of the many body shift is up to $8 \mathrm{meV}$ at room temperature at injection levels typical for laser operation, about 2.2 electrons per dot, producing a peak modal gain of $10 \mathrm{~cm}^{-1}$. The shift is smaller that the standard deviation of the Gaussian fit to the ground state absorption spectrum of $14 \mathrm{meV}$. In these samples we expect that the carrier density in the wetting layer is very small so the observed effects are due to carriers on the dots themselves.

We acknowledge financial support from the Engineering and Physical Sciences Research Council under Grant No. EP/F006683.

${ }^{1}$ P. Borri, W. Langbein, S. Schneider, U. Woggon, R. L. Sellin, D. Ouyang, and D. Bimberg, IEEE J. Sel. Top. Quantum Electron. 8, 984 (2002).

${ }^{2}$ S. Osborne, P. Blood, P. M. Smowton, J. Lutti, Y. C. Xin, A. Stintz, D. Huffaker, and L. F. Lester, IEEE J. Quantum Electron. 40, 1639 (2004).

${ }^{3}$ S. Schmitt-Rink, D. A. B. Miller, and D. S. Chemla, Phys. Rev. B 35, 8113 (1987)

${ }^{4}$ Y. Z. Hu, M. Lindberg, and S. W. Koch, Phys. Rev. B 42, 1713 (1990).

${ }^{5}$ S. V. Nair and Y. Masumoto, Phys. Status Solidi A 178, 303 (2000).

${ }^{6}$ K. Matsuda, K. Ikeda, T. Saiki, H. Saito, and K. Nishi, Appl. Phys. Lett. 83, 2250 (2003).

${ }^{7}$ R. Heitz, F. Guffarth, I. Mukhametzhanov, M. Grundmann, A. Madhukar, and D. Bimberg, Phys. Rev. B 62, 16881 (2000).

${ }^{8}$ H. C. Schneider and W. W. Chow, Phys. Rev. B 64, 115315 (2001).

${ }^{9}$ H. Y. Liu, I. R. Sellers, T. J. Badcock, D. J. Mowbray, M. S. Skolnick, K. M. Groom, M. Gutierrez, M. Hopkinson, J. S. Ng, J. P. R. David, and R. Beanland, Appl. Phys. Lett. 85, 704 (2004).

${ }^{10}$ I. O'Driscoll, P. Blood, and P. M. Smowton, IEEE J. Quantum Electron. 46, 525 (2010).

${ }^{11}$ P. Blood, G. M. Lewis, P. M. Smowton, H. Summers, J. Thompson, and J. Lutti, IEEE J. Sel. Top. Quantum Electron. 9, 1275 (2003).

${ }^{12}$ I. O'Driscoll, P. M. Smowton, and P. Blood, IEEE J. Quantum Electron. 45, 380 (2009).

${ }^{13}$ P. Blood, IEEE J. Sel. Top. Quantum Electron. 15, 808 (2009). 